\title{
A role for actin in aging and apoptosis
}

\author{
C.W. Gourlay and K.R. Ayscough ${ }^{\mathbf{1}}$
}

Department of Molecular Biology and Biotechnology, Firth Court, University of Sheffield, Western Bank, Sheffield S10 2TN, U.K.

\begin{abstract}
The actin cytoskeleton is central to many cell processes including membrane trafficking and generation of cell polarity. We have identified a role for actin in cell death and in promoting longevity of the budding yeast, Saccharomyces cerevisiae. Aging in yeast appears to occur via an apoptotic-like pathway with changes including DNA fragmentation, loss of mitochondrial membrane permeability, increase in levels of ROS (reactive oxygen species) and exposure of phosphatidylserine in the outer leaflet of the plasma membrane. This pathway can be induced by alterations in actin dynamics, such that reduced dynamics correlates with increased levels of ROS and decreased viability. Conversely, increased actin dynamics correlates with low ROS levels and increased survival. Our current studies have focused on identifying pathways which couple changes in actin dynamics to cell death.
\end{abstract}

\section{Introduction}

The yeast actin cytoskeleton is an essential structure that is organized into two major structures that can be visualized by fluorescence microscopy of rhodamine phalloidin stained cells [1]. Aligned along the mother bud axis are actin cables, bundles of long actin filaments which serve as tracks for the movement of secretory vesicles and organelles including mitochondria, peroxisomes and vacuoles [2]. The other main actin structures are the cortical actin patches. These are visualized as small dots, which are highly polarized to the buds of growing cells. Cortical actin patches are proposed to be sites of endocytosis [3]. Both actin structures are disrupted by the actin monomer-sequestering drug latrunculin-A, indicating they are highly dynamic [4]. Indeed, the dynamic state of F-actin is considered central to its ability to perform in all its known functions in yeast. Addition of actin-stabilizing drugs, or the introduction of mutations which reduce actin dynamics, both lead to decreased cell viability, indicating the importance of maintaining the dynamic stage of the actin cytoskeleton. Our investigations into the effects of altering actin dynamics have led us to demonstrate that such changes are able to modulate the level of ROS (reactive oxygen species) in a cell, and consequently to lead to cell death via an apoptoticlike pathway [5].

There are two main reasons that advances in this field have come from studies in yeast. First, there is a single actin gene (ACT1) that can be readily mutated and expressed as the sole form of actin in the cell [6]. Therefore it is possible to study the effect of single-point mutations, and there are a plethora of actin mutants that have been generated and characterized [7]. Secondly, as in other eukaryotes, the central organelle for the production of damaging ROS is the mitochondrion. However, yeast are able to grow in both fermentative and non-fermentative states, only the latter of

Key words: actin, aging, apoptosis, mitochondrion, programmed cell death, senescence. Abbreviations used: PDE2, phosphodiesterase 2; ROS, reactive oxygen species; VDAC, voltagedependent anion channel.

${ }^{1}$ To whom correspondence should be addressed (email k.ayscough@sheffield.ac.uk). which requires a functioning mitochondrion. So, defects that disrupt mitochondria, and which in many other cell types would be lethal, are open to study in this highly manipulable organism.

\section{Yeast apoptosis and aging}

Yeast normally grows as a clonal colony and each cell is surrounded by its own progeny. Each budding event leads to a birth scar on the mother and eventually this impacts on the integrity of the cell surface (the average wild-type cell can produce approx. 25 daughter cells). Daughter cells selectively inherit undamaged mitochondria [8] and receive new cellwall material [9] and so each daughter is effectively 'born' at age 0 . Continual exposure of a cell to ROS and the increasing inability to withstand ROS with age results in the accumulation of damaged cell components in mother cells. In order to preserve her own gene pool it becomes more beneficial for a mother cell to die than to utilize increasingly scarce nutrients for her own growth.

As in mammalian cells, apoptotic yeast cells show mitochondrial depolarization, high levels of ROS, DNA fragmentation and phospholipid exposure at the plasma membrane. Exposure of yeast to low levels of hydrogen peroxide, depletion of glutathione or expression of the mammalian bax gene induces apoptosis, accompanied by an increase in ROS. The role of the yeast caspase-like protein Yca1p is less clear, although there is evidence that caspase-dependent cell-death pathways are present and contribute to cell death [10]. Finally, an apoptosis-inducing factor (Aif1) is present in yeast and shares characteristics with its mammalian counterpart [11]. There are two main measures of aging in yeast: chronological aging considers the longevity of non-dividing cells (this is considered to be equivalent to post-mitotic aging in higher eukaryotes) [12]; and replicative aging is an assessment of the number of daughters generated from a mother cell [13]. In both cases, it is clear that build-up of ROS and redox regulation is a key factor in determining lifespan $[14,15]$. Importantly, deletion of superoxide dismutase or catalase genes shortens the lifespan of yeast, whereas the antioxidant 
Figure 1 Actin dynamics correlates with viability and production of ROS

Upper panels show the actin cytoskeleton stained with rhodamine-phalloidin in 5-day-old cultures of wild-type cells, act1-157 cells (exhibiting increased actin dynamics) and act1-159 cells (exhibiting reduced actin dynamics). Lower panels show, for the same strains, flow cytometric analysis to measure $\mathrm{H}_{2} \mathrm{O}_{2}$ levels as an indicator of ROS accumulation. Beneath these panels are the viabilities recorded for each strain.

Wild Type
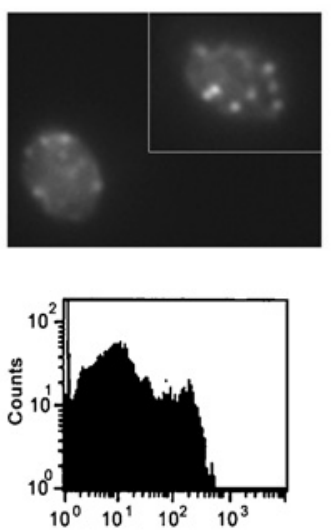

Viability act1-157
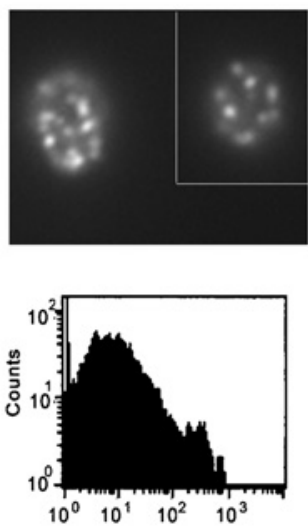

$58 \%$ act1-159
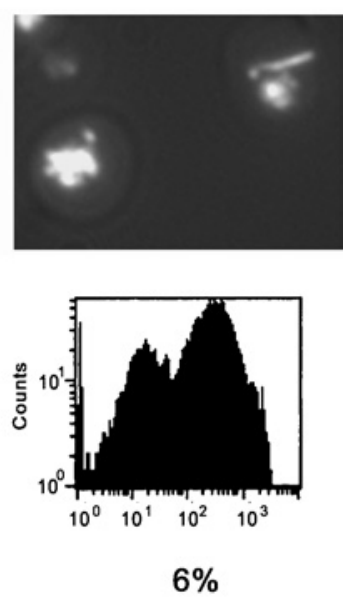

glutathione increases yeast lifespan as a result of reducing ROS levels.

\section{Evidence that actin dynamics can trigger yeast programmed cell death}

The central finding of the studies in yeast is that changes that decrease actin turnover, and that lead to the accumulation of large aggregates of F-actin, trigger an increase in the levels of ROS in the cytosol. Decreases in turnover could be induced by specific point mutations in actin that affect nucleotide exchange, by addition of the actin-stabilizing drug jasplakinolide, and in aged cells (Figure 1) [5]. Conversely, increased actin turnover, which can be induced by mutations in actin or by deletion of the gene for the actin-bundling protein Scp1p, leads to decreased production of ROS and, in turn, to increased viability [5]. Indeed, deletion of Scp1p is able to increase lifespan in both dividing and non-dividing yeast cells. Importantly, disruption of mitochondrial function prevented the accumulation of ROS in cells that exhibited reduced actin dynamics, which demonstrated that mitochondria are the source of the ROS. In addition, these cells still had aberrant actin aggregates, which showed that changes in actin in the absence of mitochondrial function do not themselves elicit the cell-death phenotype. The fact that alterations in actin dynamics can both increase and decrease levels of detectable ROS has led to the hypothesis that the actin cytoskeleton could act as a regulator for ROS release from the mitochondrion.

\section{Mechanisms coupling changes in actin dynamics to cell death}

While the results strongly support the notion that changes in actin dynamics are able to trigger an apoptotic-like path- way, the mechanism that facilitates this outcome is not known. There are a number of models that can be proposed to explain the link. Two of these are outlined here (Figures 2A and $2 \mathrm{~B}$ ). In essence, the link could be direct, i.e. actin could directly regulate interactions with the mitochondria and influence release of ROS. Alternatively, the link could be indirect and actin could be viewed as a cytosolic sensor of well-being within the cell.

\section{Direct coupling of actin to mitochondrial function}

There are known to be direct links between mitochondria and F-actin, and mitochondria can be observed to align along actin cables [16-18]. In addition, disruption of actin cables results in altered mitochondrial morphology. Mitochondria are also known to use actin cables as tracks to facilitate inheritance by the daughter cell. Indeed, it has generally been thought that the main reason for the association between actin and mitochondria is to ensure movement into the new bud. One interesting recent observation is that daughter cells inherit newly synthesized mitochondria, while mother cells retain older, often damaged mitochondria [19] suggesting that there could be selective interaction and movement of different domains of the mitochondria. One mechanism that would allow direct coupling of ROS release to actin function is by regulation of opening and closing of the VDACs (voltage-dependent anion channels; Figure 2A). These are mitochondrial membrane pores which regulate the release of proapoptotic factors such as cytochrome $c$, and which are important for maintaining the mitochondrial membrane potential. Certainly, mammalian gelsolin, an actin regulatory protein, is able to affect entry to apoptosis by its effects on VDACs (see below). Interestingly, evidence also exists to suggest that actin regulates VDAC closure in the filamentous 
Figure 2 | Models for coupling actin dynamics to apoptosis

(A) Direct regulation of ion channels. Results indicate that actin can be involved in the regulation of the VDAC, which has an important role in apoptotic cell death. Opening and closure of the VDAC is involved in regulating the permeability of the mitochondrial outer membrane. In an open, or proapoptotic, state, which occurs when the actin cytoskeleton is stabilized, the VDAC allows the release of apoptogenic proteins, such as cytochrome $c$, from the mitochondria. Closure of the VDAC, in response to a dynamic actin cytoskeleton, has been demonstrated to confer resistance to apoptotic stimuli. (B) Actin as a sensor of cell well-being. The Ras/cAMP pathway is highly conserved amongst eukaryotes. In yeast cells, the small G-protein Ras2, a homologue of mammalian oncogene Ras, regulates adenylate cyclase (Cyг1) that in turn catalyses synthesis of CAMP from ATP. CAMP then activates protein kinase A (PKA), which leads to the transcription of stress response genes. In healthy cells, repression of the Ras/cAMP pathway on exposure to stress appears to trigger remodelling of actin structures, which is proposed to be important for survival. Cells with aggregated F-actin appear to constitutively activate the Ras/cAMP pathway, which leads to elevated CAMP levels, mitochondrial dysfunction, ROS accumulation and cell death. In this situation, the Ras pathway no longer appears to be able to trigger appropriate actin remodelling, suggesting a block in the normal stress response pathway required to protect actin. Overexpression of PDE2 that decreases CAMP levels is able to rescue the actin clumping and also restores ROS to wild-type levels.

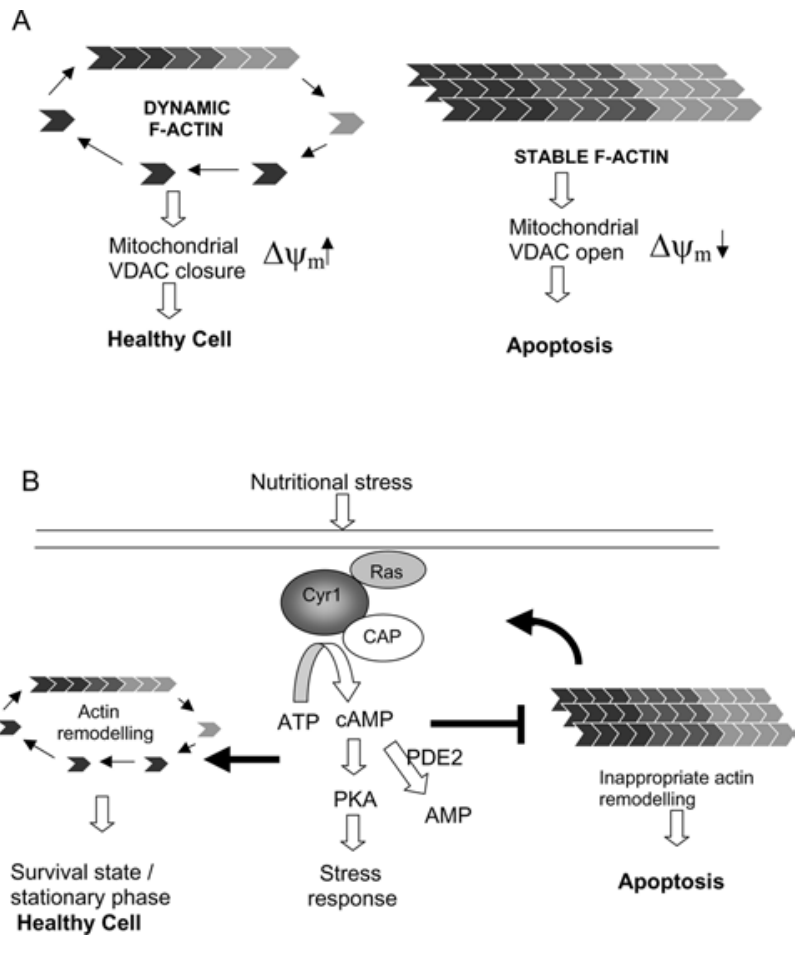

fungus, Neurospora crassa [20]. In purified mitochondria from $N$. crassa, addition of an actin-stabilizing drug, phalloidin, caused prolonged opening of VDAC pores and an increase in released ROS, suggesting that actin has a conserved role in cell death by regulating VDAC in eukaryotic cells.

\section{Stress signalling, actin and apoptosis: actin dynamics as a sensor of cell well-being}

Remodelling of the cytoskeleton in response to stress is a fundamental process in many eukaryotic cells. As a less dynamic cytoskeleton is unable to respond effectively to stress, it would be prudent in a population to remove cells with this non-responsive cytoskeleton (either in a tissue or in a colony of unicellular eukaryotes). Such a scenario requires that cells are able to couple a signalling pathway involved in cell viability to remodelling of the actin cytoskeleton, such that the pathway both regulates, and is regulated by, actin dynamics (Figure 2B). This pathway would determine whether a cell is maintained in a population by using actin dynamics as a biosensor of health. A recognized example of a pathway fulfilling these requirements is the Ras/cAMP pathway in Saccharomyces cerevisiae. In yeast, this pathway is a regulator of longevity and its negative regulation (repression) is essential for the response to environmental stresses [21]. Constitutive activation of the Ras pathway leads to a failure to up-regulate proteins that protect against oxidative stress, to mitochondrial dysfunction and to a reduced lifespan. An increase in lifespan has been noted in cells lacking Ras, as they have elevated levels of proteins that are involved in protecting against oxidative stress. The Ras pathway has also been shown to play a role in actin regulation [22]. Recent results support this idea and have revealed that the Ras/cAMP pathway and actin are able to interact to regulate oxidative stress and cell viability [23]. Cells that lack either of the two actin regulatory proteins, End3p or Sla1p, form large aberrant F-actin aggregates and also display phenotypes associated with overactivation of the Ras/cAMP signalling cascade. These include mitochondrial dysfunction, elevated ROS levels, reduced longevity and a failure to respond to nutritional stress. In support of Ras/cAMP overactivation as the cause of oxidative stress and cell death in these actin regulatory mutants, overexpression of PDE2 (phosphodiesterase 2), a negative regulator of the Ras/cAMP pathway, can reduce ROS levels and restore viability to wild-type levels [23]. Therefore the activity of the Ras/cAMP pathway is responsive to the dynamic state of the actin cytoskeleton in yeast. In turn, overexpression of PDE2 in these mutant cells alleviates the formation of actin aggregates indicating that the Ras/cAMP pathway also regulates actin dynamics. This result supports a cross-talk mechanism between the Ras/cAMP signalling pathway and the actin cytoskeleton, which modulates cell survival in yeast. Thus, less energetic, aged or mutated cells, which exhibit reduced cytoskeletal dynamics, cannot function to feed signals back to the Ras pathway, and would be eliminated from the population. Current studies are focusing on identifying further elements of this cell-death pathway.

\section{Actin, apoptosis and aging in mammalian cells}

Although the evidence from yeast studies clearly shows that changes in actin can induce release of ROS from 
mitochondria, the case in mammalian cells is less clear and some results present a conflicting picture. Part of the controversy might lie in the diverse range of cell types that have been investigated, and the assays used to detect cellular changes. Other complications include the fact that several actin isoforms are expressed, a lack of available actin mutants and that it is difficult to readily abrogate mitochondrial function. However, there is now a significant amount of circumstantial evidence that indicates a role for actin in triggering cell death and, potentially, aging, and that this function can lie upstream of mitochondrial ROS release.

A link between actin stabilization and aging is suggested by the finding that several screens to identify biomarkers of aging in mammalian cells have identified the SM22 protein [24-26]. SM22, which is also known as transgelin, cross-links actin filaments in vitro and is the mammalian homologue of yeast Scp1p [27,28]. Just as studies in yeast have shown that elevated Scp1p can cause inappropriate actin clumping and cell death, so studies in mammalian cells have observed that senescent cells have higher levels of SM22/transgelin. Additionally, reduced levels of SM22 have been reported in several tumour cell lines [29]. Further studies on yeast and mammalian cells might now shed light on the process of cell aging and, in particular, how this process is linked to the normal apoptotic pathway of cell death.

Some of the clearest results supporting links between actin and apoptosis pathways in mammalian cells come from studies using drugs that affect actin turnover. As in yeast, the addition of jasplakinolide induces very robust actin stabilization and rapidly leads to the accumulation of large F-actin aggregates. Concomitant with this reduction in actin turnover is the induction of a cell-death pathway $[30,31]$. While there might be some variability between cell types as to whether actin changes alone can induce apoptosis or whether there need to be additional stimuli, it is clear that, as in yeast, actin stabilization can induce cell death and this is mediated through changes involving mitochondria.

The best-studied physiological regulator of actin that also has a role in apoptosis is gelsolin. This protein is well characterized in mediating actin reorganization in response to changes in $\mathrm{Ca}^{2+}$ and phosphoinositides in vivo and has a demonstrated role in cell motility [32]. Overexpression of gelsolin has been shown to inhibit apoptosis in Jurkat cell lines in response to conditions that otherwise induce cell death [33]. Further investigation has revealed that gelsolin-mediated inhibition of apoptosis occurs by blocking the loss of mitochondrial membrane potential and also by preventing caspase activation. A fraction of gelsolin co-localizes with mitochondria, indicating that its role in regulating the mitochondrial potential might occur through a directbinding mechanism [34]. Recent studies using neuronal cells have correlated the anti-apoptotic role of gelsolin with its dynamic actions on the actin cytoskeleton, and have demonstrated that the key role of gelsolin in protection from apoptosis is its ability to enhance actin depolymerization [35]. Overexpression of gelsolin is likely to increase the turnover of actin and reduce the overall stability of the actin cytoskeleton, which makes the situation analagous to the effect of the scp1 deletion or the actin-destabilizing mutation (act1-157) in yeast [5].

\section{Summary}

The actin cytoskeleton is known to be crucial in mediating cell responses to both internal and external signals. Central to its function is its dynamic structure and its ability to be reorganized into distinct structures that are suitable for particular tasks within the cell. Defects in the ability to regulate the dynamics of actin are therefore likely to have detrimental effects on cell function. Therefore it may not be surprising that a cell that has lost the ability to signal to, and reorganize, its actin experiences a signal for cell death. The exact role for actin in the pathway remains unclear. Whether actin itself can regulate channels in the mitochondria and directly regulate the onset of apoptosis, or whether the effect of actin aggregation is sensed by the mitochondria through indirect pathways, are two questions under investigation.

K.R.A. and C.W.G. are supported by a Medical Research Council (MRC) senior non-clinical research fellowship to K.R.A. (G117/394).

\section{References}

1 Adams, A.E.M. and Pringle, J.R. (1984) J. Cell Biol. 98, 934-945

2 Pruyne, D., Legesse-Miller, A., Gao, L., Dong, Y. and Bretscher, A. (2004) Annu. Rev. Cell Dev. Biol. 20, 559-591

3 Kaksonen, M., Sun, Y. and Drubin, D.G. (2003) Cell (Cambridge, Mass.) 115, 475-487

4 Ayscough, K.R., Stryker, J., Pokala, N., Sanders, M., Crews, P. and Drubin, D.G. (1997) J. Cell Biol. 137, 399-416

5 Gourlay, C.W., Carpp, L.N., Timpson, P., Winder, S.J. and Ayscough, K.R. (2004) J. Cell Biol. 164, 803-809

6 Ayscough, K.R. and Drubin, D.G. (1996) Annu. Rev. Cell Dev. Biol. 12 129-160

7 Wertman, K.F., Drubin, D.G. and Botstein, D. (1992) Genetics 132 337-350

8 Lai, C.-Y., Jaruga, E., Borghouts, C. and Jazwinski, S.M. (2002) Genetics 162, 73-87

9 Smeal, T., Claus, J., Kennedy, B., Cole, F. and Guarente, L. (1996) Cell (Cambridge, Mass.) 84, 633-642

10 Madeo, F., Herker, E., Maldener, C., Wissing, S., Lachelt, S., Herlan, M., Fehr, M., Lauber, K., Sigrist, S.J., Wesselborg, S. et al. (2002) Mol. Cell 9 911-917

11 Wissing, S., Ludovico, P., Herker, E., Buttner, S., Engelhardt, S.M., Decker, T., Link, A., Proksch, A., Rodrigues, F., Corte-Real, M. et al. (2004) J. Cell Biol. 166, 969-974

12 Maclean, M., Harris, N. and Piper, P.W. (2001) Yeast 18, 499-509

13 Tissenbaum, H. and Guarente, L. (2002) Dev. Cell 1, 9-19

14 Fabrizio, P., Battistella, L., Vardavas, R., Gattazzo, C., Liou, L.L., Diaspro, A., Dossen, J.W., Gralla, E.B. and Longo, V.D. (2004) J. Cell Biol. 166, 1055-1067

15 Herker, E., Jungwirth, H., Lehmann, K.A., Maldener, C., Frohlich, K.-U., Wissing, S., Buttner, S., Fehr, M., Sigrist, S. and Madeo, F. (2004) l. Cell Biol. 164, 501-507

16 Boldogh, I., Vojtov, N., Karmon, S. and Pon, L.A. (1998) J. Cell Biol. 141 1371-1381

17 Read, E.B., Okamura, H.H. and Drubin, D.G. (1992) Mol. Biol. Cell 3 , 429-444

18 Simon, V.R., Karmon, S.L. and Pon, L.A. (1997) Cell Motil. Cytoskeleton 37, 199-210

19 Aguilaniu, H., Gustafsson, L., Rigoulet, M. and Nyström, T. (2003) Science 299, 1751-1753

20 XU, X., Forbes, J.G. and Colombini, M. (2001) J. Membr. Biol. 180, 73-81

21 Rolland, F., Winderickx, J. and Thevelein, J.M. (2002) FEMS Yeast Res. 2, 183-201 
$22 \mathrm{Ho}$, J. and Bretscher, A. (2001) Mol. Biol. Cell 12, 1541-1555

23 Gourlay, C.W. and Ayscough, K.R. (2005) J. Cell Sci. 118, 2119-2132

24 Dumont, P., Burton, M., Chen, Q.M., Gonos, E.S., Frippiat, C., Mazarati, J.B., Eliaers, F., Remacle, J. and Toussaint, 0. (2000) Free Radical Biol. Med. 28, 361-373

25 Gonos, E.S., Derventzi, A., Kveiborg, M., Agiostratidou, G., Kassem, M. Clark, B.F.C., Jat, P.S. and Rattan, S.I.S. (1998) Exp. Cell Res. 240, 66-74

26 Thweatt, R., Lumpkin, C.K. and Goldstein, S. (1992) Biochem. Biophys. Res. Commun. 187, 1-7

27 Camoretti-Mercado, B., Forsythe, S.M., LeBeau, M.M., Espinosa, R., Vieira, J.E., Halayko, A.J., Willadsen, S., Kurtz, B., Ober, C., Evans, G.A. et al. (1998) Genomics 49, 452-457

28 Prinjha, R.K., Shapland, C.E., Hsuan, J.J., Totty, N.F., Mason, I.J. and Lawson, D. (1994) Cell Motil. Cytoskeleton 28, 243-255

29 Lawson, D., Harrison, M. and Shapland, C. (1997) Cell Motil. Cytoskeleton 38, $250-257$
30 Odaka, C., Sanders, M.L. and Crews, P. (2000) Clin. Diagn. Lab. Immunol. 7, 947-952

31 Posey, S. and Bierer, B. (1999) J. Biol. Chem. 274, 4259-4265

32 Kwiatkowski, D.J. (1999) Curr. Opin. Cell Biol. 11, 103-108

33 Ohtsu, M., Sakai, N., Fujita, H., Kashiwagi, M., Gasa, S., Shimizu, S., Eguchi, Y., Tsujimoto, Y., Sakiyama, Y., Kobayashi, K. et al. (1997) EMBO J. 16, $4650-4656$

34 Koya, R.C., Fujita, H., Shimizu, S., Ohtsu, M. Takimoto, M., Tsujimoto, Y. and Kuzumaki, N. (2000) J. Biol. Chem. 275, 15343-15349

35 Harms, C., Bosel, J., Lautenschlager, M., Harms, U., Braun, J.S., Hortnagl, H., Dirnagl, U., Kwiatkowski, D.J., Fink, K. and Endres, M. (2004) Mol. Cell. Neurosci. 25, 69-82

Received 10 June 2005 